\title{
Hemophagocytic Histiocytic Sarcoma in Dog
}

\author{
Juliana das Chagas Goulart', Felipe Jacques Sanches ${ }^{2}$, Natalie Bertelis Merlini ${ }^{3}$, Barbara Cristina Mazzucatto ${ }^{4}$, \\ Beatriz Gasser ${ }^{5} \&$ Paulo Fernandes Marcusso $^{6}$
}

\begin{abstract}
Background: Histiocytic sarcoma can present in a localized or disseminated form, being a malignant neoplasm derived from interstitial dendritic cells. Another form of the disease is called hemophagocytic histiocystic sarcoma (HHS), which originates from macrophages located in the splenic red pulp and bone marrow. HHS is a rare neoplasm with unknown etiology and rapid development. It mainly affects middle-aged to elderly animals. It primarily affects the spleen and bone marrow, with occurence of metastases in other organs. Some canine breeds are considered more predisposed to this neoplasia, among them the Rottweiler. The aim of this study was to report a case of HHS in a Rottweiler dog.

Case: An 8-year-old Rottweiler suffering from hyporexia and progressive weight loss for a month was attended at the Veterinary Hospital of State University of Maringá. On physical examination, he only presented mild discomfort on abdominal palpation. Blood count was performed, which revealed mild thrombocytopenia $(145,000 / \mu \mathrm{L}$, reference value for the species 150,000-500,000/ $\mu \mathrm{L}$ ). The Polymerase Chain Reaction (PCR) exam was requested for babesiosis and ehrlichiosis, due to a history of ectoparasites. The animal was released with the prescription of Doxycycline at a dose of 10 $\mathrm{mg} / \mathrm{kg}$ orally for 28 days. After 5 days, the condition worsened and the animal returned with emesis, apathy and hyporexia, being hospitalized. Abdominal ultrasound, blood count, urinalysis, serum urea and creatinine tests were requested. Among the hematological changes, thrombocytopenia $(116,000 / \mu \mathrm{L})$ and the appearing of regenerative anemia and atypical round cells with ample and slightly basophilic cytoplasm in the smear, similar to histiocytes, stood out. On ultrasound examination, splenomegaly was observed with hypoechogenic areas. Ultrasound-guided fine-needle aspiration cytology (FNAC) of the spleen was performed, which revealed the presence of abundant cellularity consisting of round cells arranged individually and in small groups, with variable nucleus / cytoplasm ratio, slightly basophilic cytoplasm and, sometimes, vacuolized. Eccentric nucleus with chromatin ranging from loose to dense, with evident and often multiple nucleus. In addition, a large number of multinucleated giant cells, mitosis figures (sometimes atypical) and intense anisocytosis and anisocariosis were observed. Furthermore, it was possible to observe erythrophagocytic activity, alterations compatible with histiocytic sarcoma (HS). After 10 days of the first visit, the animal died. At necropsy, samples from altered areas of the spleen, lungs and lymph nodes were collected and sent for histopathological and immunohistochemical examination, in which the diagnosis of hemophagic histiocytic sarcoma was confirmed, in addition to lungs and lymph node metastasis. Discussion: The prognosis of HHS is the worst among histiocytic disorders, as it is associated with coagulopathies and severe anemia due to erythrophagocytic activity, as occurred in the case presented, with a maximum survival time between one to two months. The described animal died ten days after showing the first clinical signs. The immunohistochemistry technique using HLA-DR, CD18, CD163, and CD11d markers was essential to confirm the cell line. Positivity for CD11d marker was found, which allowed to classify the neoplasm as hemophagocytic HS. This report exposes the importance of cytological examination for screening and histopathological and immunohistochemical tests to confirm the diagnosis of this extremely aggressive neoplasm.
\end{abstract}

Keywords: Neoplasms, hematology, cytology, histopathology, immunohistochemistry. 


\section{INTRODUCTION}

Hemophagic or hemophagocytic histiocytic sarcoma (HHS) is currently the only histiocytic disorder described in dogs and cats that originates from macrophages located in the splenic red pulp and bone marrow $[9,10]$. Unlike dendritic cells neoplasms, the hemophagocytic form does not develop distinct masses, but results in diffuse hepatomegaly and splenomegaly [5].

Histiocytic sarcomas are uncommon in domestic dog population, however there is a high incidence in breeds such as Bernese Mountain Dog, Flat-coated Retriever, Golden Retriever, Rottweiler and Labrador Retriever, which suggests hereditary risk factors $[7,10]$. Cytogenetic analysis demonstrated the loss of several tumor suppressor genes in cases of canine histiocytic sarcoma $[3,7]$.

The hemophagocytic type of histiocytic sarcoma is a rare neoplasm, of unknown etiology and rapid development. It initially affects the spleen and bone marrow, forming metastases in other organs such as liver and lungs. The histiocytic sarcoma complex affects mainly middle-aged to elderly animals, although there are reports in young animals and there is no sexual predisposition $[1,3-5,11]$. This paper aims to report a rare case of histiocytic hemophagocytic sarcoma in a Rottweiler dog.

\section{CASE}

An 8-year-old male Rottweiler, weighing $46.2 \mathrm{~kg}$, arrived at the Veterinary Hospital of the State University of Maringá - Umuarama Campus, presenting hypoxia and progressive weight loss for a month. On physical examination, he only presented mild discomfort on abdominal palpation. A blood count was requested, which showed mild thrombocytopenia $(145,000 / \mu \mathrm{L})$.

After this result, the Polymerase Chain Reaction (PCR) exam was requested to investigate babesiosis and ehrlichiosis, since the occurrence of hemoparasitosis is common in the region and there was a history of ectoparasites. The animal was released and Doxycycline ${ }^{1}$ was prescribed at a dose of $10 \mathrm{mg} /$ $\mathrm{kg}$ orally for 28 days, as a therapeutic diagnosis while the PCR result was not available.

After 5 days, the animal returned with clinical worsening, presenting emesis, apathy and hyporexia, and was hospitalized. Abdominal ultrasound, blood count, urinalysis, serum urea and creatinine tests were requested.
In the second blood count (Table 1), red blood cells with mild anisocytosis and polychromasia, neutrophilic leukocytosis with regenerative left shift, worsening thrombocytopenia and a population of round cells not classified in the tail of the blood smear, which were characterized by being round with abundant cytoplasm, and slightly basophilic, eccentric round to oval nucleus with finely punctiform chromatin, similar to histiocytes were found.

The serum urea $(65 \mathrm{mg} / \mathrm{dL})$ and creatinine $(1.8$ $\mathrm{mg} / \mathrm{dL}$ ) values were above the reference values for the species (reference: $21.4-59.92 \mathrm{mg} / \mathrm{dL}$ and $0.5-1.5 \mathrm{mg}$ / $\mathrm{dL}$, respectively) and urinalysis revealed high density (> 1.040), proteinuria (100 mg/dL) and moderate presence of hyaline and granular cylinders. The ultrasound examination revealed splenomegaly and the presence of two hypoechogenic rounded structures in the splenic parenchyma, one measuring $0.71 \mathrm{~cm} \times 0.52 \mathrm{~cm}$ and the other $0.77 \mathrm{~cm} \times 0.6 \mathrm{~cm}$. Due to the presence of these structures in the spleen, ultrasound-guided fine-needle aspiration cytology (FNAC) was performed.

Cytology revealed the existence of an abundant cellularity consisting of round cells arranged individually and in small groups, with a variable nucleus / cytoplasm ratio, slightly basophilic cytoplasm and sometimes vacuolated. Eccentric nucleus with chromatin ranging from loose to dense, with evident and often multiple nucleus. In addition, a large number of multinucleated giant cells, mitosis figures (sometimes atypical) and intense anisocytosis and anisocariosis were observed (Figure 1). Furthermore, it was possible to observe erythrophagocytic activity (Figure 2). Such characteristics are suggestive of histiocytic sarcoma, requiring histopathology for confirmation and immunophenotyping to characterize the neoplasm.

In view of the FNAC result, the owner opted to perform palliative treatment at home, with omeprazole ${ }^{2}$, ranitidine $^{2}$ and dipyrone ${ }^{2}$. After 2 days, the condition worsened and the animal returned with musculoskeletal weakness, anorexia, apathy, tachypnea, oligodipsia and hematuria. In a new blood test, there was worsening of thrombocytopenia and anemia, with moderate hypochromia, mild anisocytosis and polychromia of the red blood cells, in addition to an increase in neutrophilic leukocytosis with left shift (Table 1) and an increase in atypical round cells in the blood smear. During this period, the PCR results came out, which was negative for Erlichia sp. and Babesia sp. 


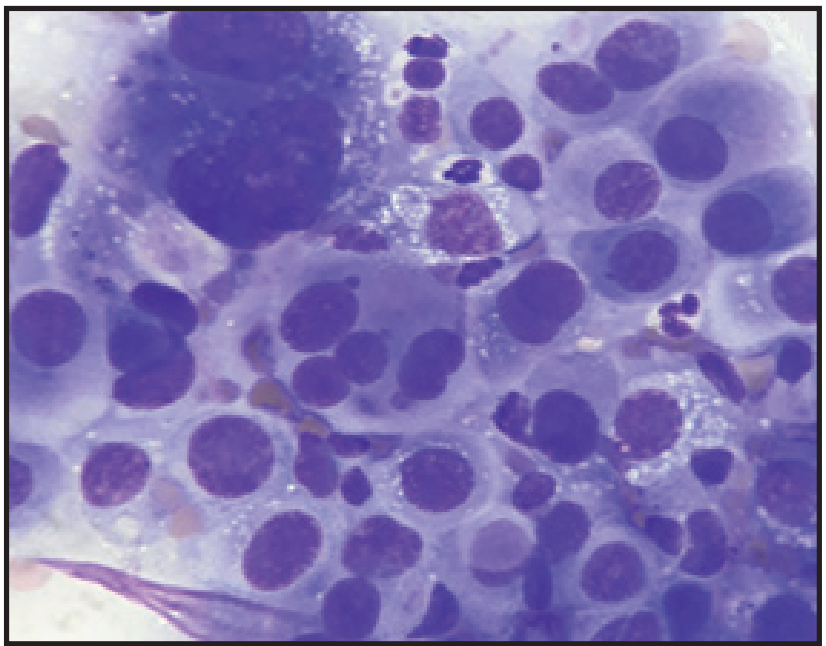

Figure 1. The predominant population consists of round pleomorphic cells that were often multinucleated. Expressive anisocariosis and anisocytosis [Fast panotic; Obj.100x].

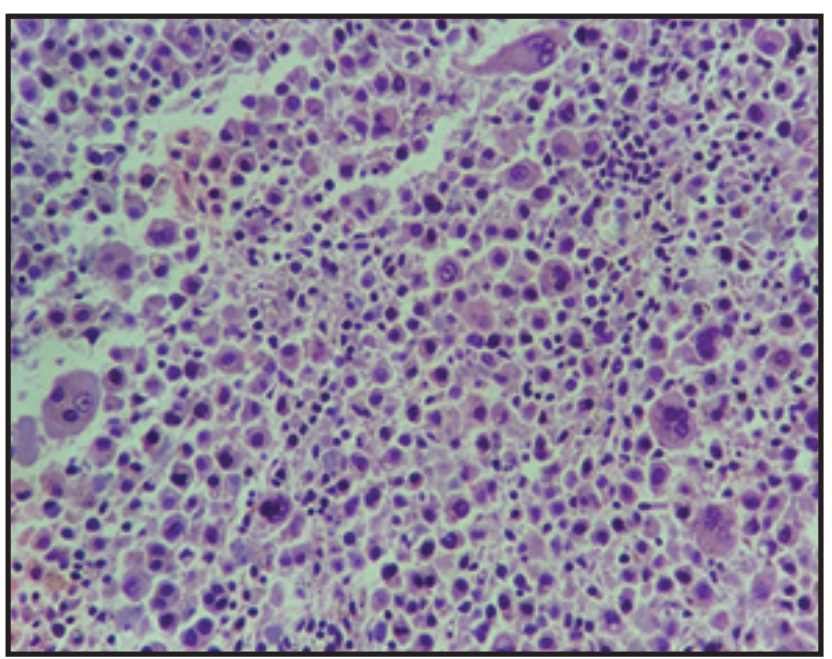

Figure 3. Histological section of the spleen showing diffuse neoplastic cells in the organ. Giant, multinucleated cells with moderate anisocytosis and anisocariosis are observed [HE; Obj.40x].

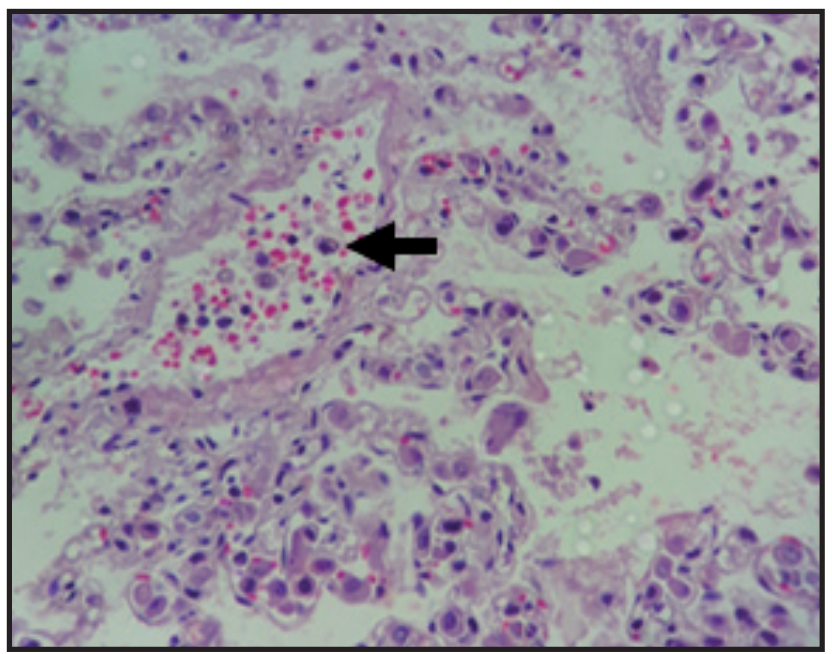

Figure 5. Lung fragment showing an intense amount of neoplastic cells in the parenchyma and invading the blood vessel (arrow) [HE; Obj.40x].

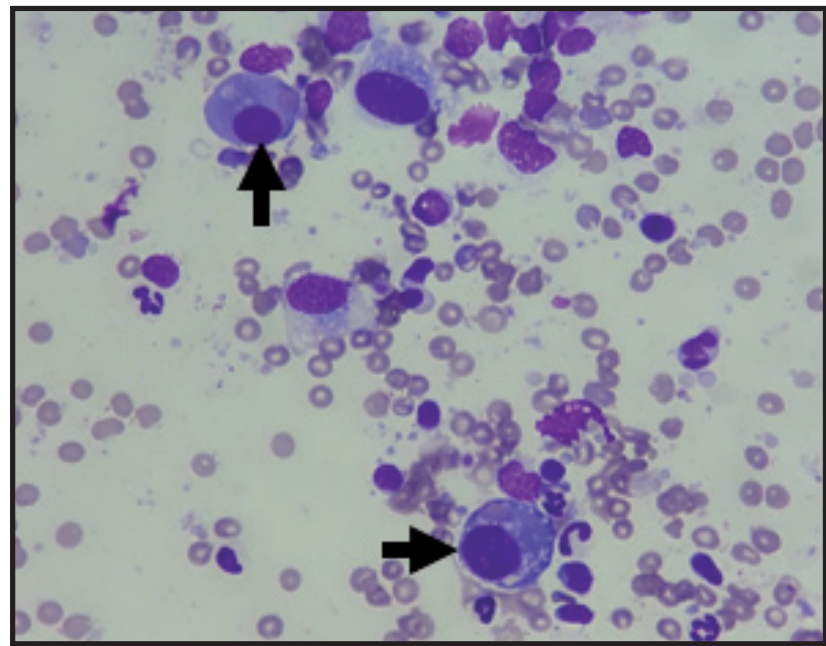

Figure 2. Fine-needle aspiration of the splenic nodule. Neoplastic cells showing erythrophagocytosis (arrows) [Fast panotic; Obj.100x].

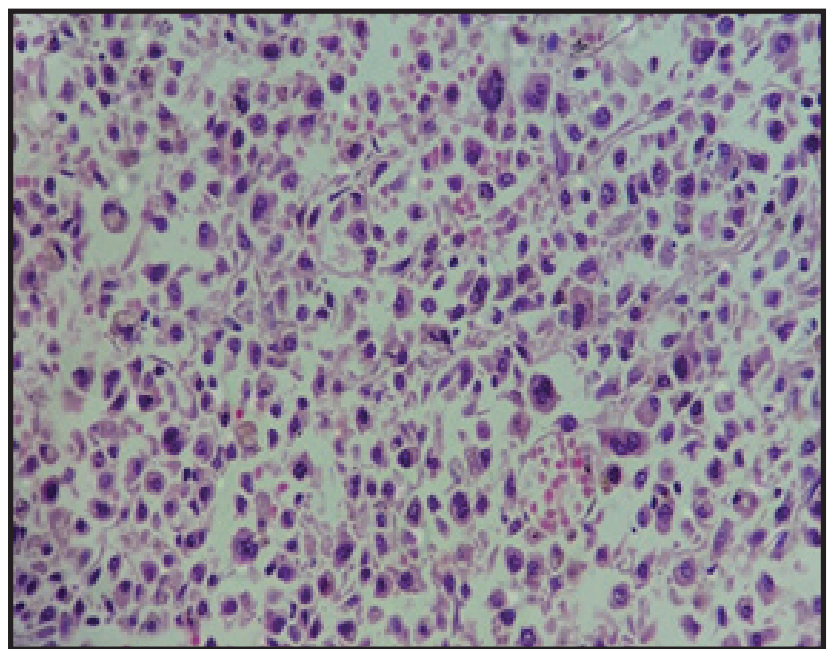

Figure 4. Histological section of the lymph node showing scattered neoplastic cells in the lymphoid tissue [HE; Obj.40x].

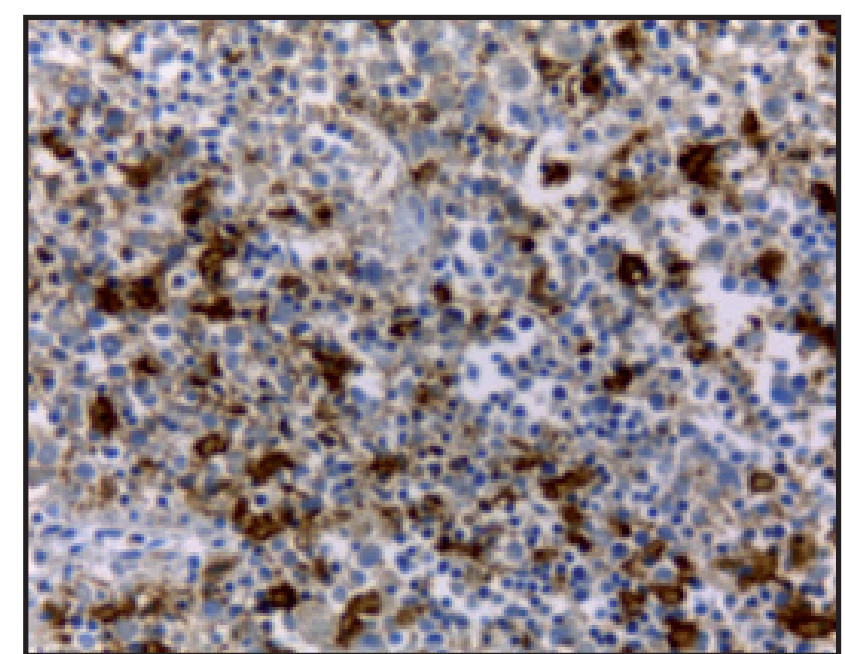

Figure 6. Photomicrograph of the spleen with CD11d positive cytoplasmic markings that identifies the macrophages origin [Obj.40x]. 


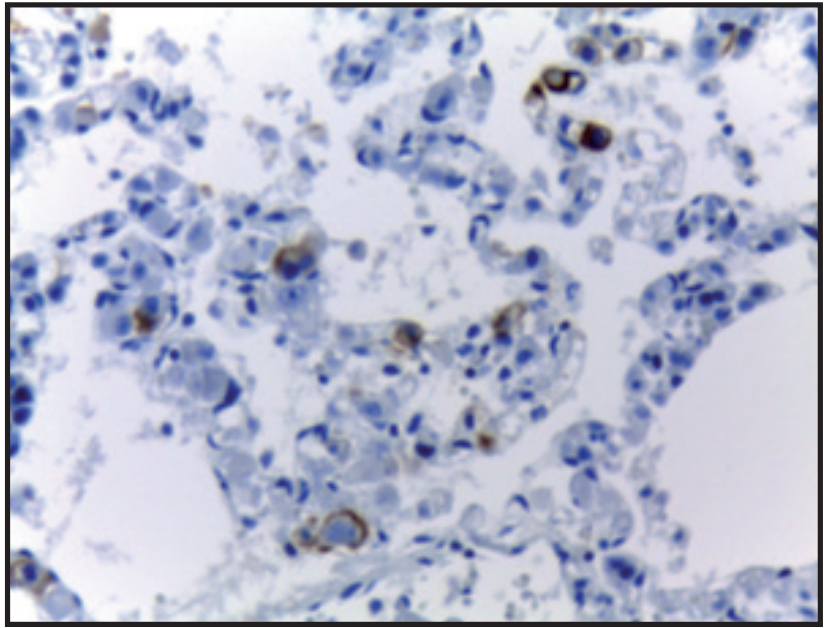

Figure 7. Photomicrograph of the lung showing positive cells with CD11d citoplasmic markings [Obj.40x].
The animal worsened and died three days after hospitalization. At necropsy, splenomegaly with diffuse pallor was observed, as well as pale areas in the lung and mesenteric and mediastinal lymph nodes. Samples of these organs were collected for histopathological evaluation.

The morphological diagnosis of the histopathological examination of the fragment of the spleen (Figure 3) confirmed the FNAC suggestive diagnosis and the fragments of lymph nodes (Figure 4) and lung (Figure 5) confirmed histiocytic sarcoma metastasis.

To discover the cell line and make the definitive diagnosis, an immunohistochemistry exam was requested, using the marker panel for histiocytic neoplasms:

Table 1. Second and Last blood count results from an 8-year-old Rottweiler with hemophagocytic histiocystic sarcoma.

\begin{tabular}{ccccc}
\hline Analyte & Second blood & Last blood & Unity & Reference \\
\hline Red Blood Cells & 5.06 & 3.89 & $\mathrm{x} 10^{6} / \mu \mathrm{L}$ & $5.5-8.5$ \\
Hemoglobin & 12.5 & 8.6 & $\mathrm{~g} / \mathrm{dL}$ & $12-18$ \\
Hematocrit & 33.5 & 26 & $\%$ & $37-55$ \\
MCV & 66.1 & 66.8 & $\mathrm{fL}$ & $60-77$ \\
MCHC & 36 & 33.1 & $\mathrm{~g} / \mathrm{dL}$ & $32-36$ \\
Metarubricytes & 1 & 2 & $\%$ & 0 \\
TPP & 5.6 & 5.0 & $\mathrm{~g} / \mathrm{dL}$ & $6.0-8.0$ \\
Total Leukocytes & 21,186 & 24,402 & $/ \mu \mathrm{L}$ & $6,000-17,000$ \\
Bands & 1,271 & 1,464 & $/ \mu \mathrm{L}$ & $0-300$ \\
Neutrophils & 17,161 & 20,742 & $/ \mu \mathrm{L}$ & $3,000-11,500$ \\
Lymphocytes & 1,059 & 1,220 & $/ \mu \mathrm{L}$ & $1,000-4,800$ \\
Monocytes & 1,271 & 732 & $/ \mu \mathrm{L}$ & $150-1,350$ \\
Eosinophils & 424 & 244 & $/ \mu \mathrm{L}$ & $100-1,250$ \\
Platelets & 116,000 & 78,000 & $/ \mu \mathrm{L}$ & $150,000-500,000$ \\
\hline
\end{tabular}

$\mathrm{MCV}=$ Mean Corpuscular Volume; $\mathrm{CHCM}=$ Mean Corpuscular Hemoglobin Concentration; TPP= Total Plasma Protein.

Table 2. Results of the histiocytic neoplasms marker panel from an 8-year-old Rottweiler with hemophagocytic histiocystic sarcoma.

\begin{tabular}{cccc}
\hline Antibodies & Clone & Results \\
\hline CD11d & Hemophagocytic Histiocytic Sarcoma & CA12.10C12 & $\begin{array}{c}\text { Positive in neoplastic cells (Spleen, } \\
\text { lung and lymph node) }\end{array}$ \\
HLA-DR & Main Histocompatibility Complex & TAL.1B5 & Positive in neoplastic cells (Spleen) \\
& MHC II & & \\
CD18 & Histiocytic neoplasms Marker & CA16.3C10 & Positive in neoplastic cells (Spleen) \\
CD163 & Histiocytic neoplasms and leukemias & Polyclonal & Positive in neoplastic cells (Spleen) \\
& Marker & & \\
CD3 & Epsilon chain T lymphocyte Receptor & Polyclonal & Negative in neoplastic cells (Spleen) \\
CD20 & B Lymphoid lineage Marker & Polyclonal & Negative in neoplastic cells (Spleen) \\
\hline
\end{tabular}


HLA-DR ${ }^{3}, \mathrm{CD}_{1} 8^{3}, \mathrm{CD}_{163}{ }^{3}, \mathrm{CD}^{3}, \mathrm{CD} 20^{3}$ and $\mathrm{CD} 11 \mathrm{~d}^{3}$, being the later specific for macrophages residing in hematopoietic sites, such as splenic red pulp and bone marrow (Figure 4). The result confirmed the diagnosis of histiocytic sarcoma of hemophagocytic type.

\section{DISCUSSION}

HHS is a rare neoplasm, of unknown etiology and rapid development. Its prognosis is the worst among histiocytic disorders, as it is associated with coagulopathies and severe anemia due to erythrophagocytic activity, with a maximum survival time between one to two months [4]. In contrast, in a study by Moore et al. [10] with 17 dogs with HHS, the survival time ranged from two to 32 weeks. In this report, the patient died 10 days after the suggestive diagnosis of the cytopathological examination. In cases of HHS, the animal is often euthanized at the time of diagnosis due to the poor health conditions he is presented and the poor prognosis [6].

Several characteristics stated in this case corroborate the literature, where the rottweiler is reported as one of the most predisposed breeds to this neoplasm, in addition to preferentially affecting middle-aged to elderly animals [2-4,10]. Furthermore, in Rottweilers, Liao et al. [8] detected a high frequency of mutation in the MET proto-oncogene (coding for tyrosine kinase), which may contribute to the breed's predisposition to neoplasms, including histiocytic sarcoma.

Animals with HHS show nonspecific clinical signs, such as lethargy, weight loss and pale mucous membranes, the same signs reported in the present case. The occurrence of splenomegaly with the presence of hypoechogenic rounded structures in the presented case differs from the literature, where splenomegaly is generally reported with no visible nodules in the splenic parenchyma or in metastatic sites such as lung and liver, sometimes hepatomegaly is observed $[2,4,10]$. Thus, after the FNAC result, the presence of nodules in the spleen suggested the primary impairment of this organ.

The hematological changes of the evaluated dog showed an evolution consistent with the literature, where the development and worsening of anemia, thrombocytopenia and hypoproteinemia were observed. In addition, there are also reports of prolongation of thromboplastin partial time, hypoalbuminemia, hypocholesterolemia and hyperbilirubinemia $[2,4,10]$.
Albumin was not measured, however, the hypoproteinemia observed suggests that it is due to hypoalbuminemia. Regenerative anemia, thrombocytopenia and hypoproteinemia agree with the results presented by Moore et al. [10], where $94 \%, 88 \%$ and $82 \%$ of dogs showed these changes, respectively. Many studies have demonstrated the presence of anemia in patients with histiocytic tumors, both related to erythrophagocytosis by neoplastic cells, and due to hypoplasia and infiltration of neoplastic histiocytes in the bone marrow [5].

Serum urea and creatinine presented slightly above the reference values for the species, added to the high urinary density, indicate a pre-renal azotemia, due to dehydration caused by emesis and oligodipsia. However, the moderate presence of cylinders and proteinuria indicate a probable acute kidney injury, and that the persistence of azotemia led to renal azotemia.

In FNAC, the erythrophagocytic activity observed was decisive for suggesting the diagnosis. Even in cases where histiocytes are well differentiated, the disease $>\mathrm{s}$ behavior is aggressive, with a high degree of metastasis in the liver, lung and lymph nodes, the latter occurring less frequently, in addition to the appearance of neoplastic cells in the blood smear [9].

At necropsy, macroscopic changes were observed in the spleen, lung and lymph nodes, where samples were collected and sent for histopathological examination, confirming the cytological examination results and detecting lung and lymph node metastasis. The lesions observed in the spleen and lung agree with those presented by Moore et al. [10]. These authors also observed neoplastic infiltration in the liver, bone marrow and lymph nodes of five of the eleven dogs affected. In the present case, it was not possible to perform bone marrow collection.

The diagnosis is based on the morphology of the tumor cells through cytology and histology, however the definitive diagnosis is obtained through immunophenotyping by searching for the CD1a, CD11c / CD18 markers (originated in dendritic cells) or CD11d / CD18 markers (originated in macrophages) [2]. CD11d being expressed exclusively by macrophages present in splenic red pulp and bone marrow. Immunohistochemistry for CD18 markers is diffusely positive in all histiocytic tumors [3].

The immunohistochemistry technique allowed the establishment of the histiocytic origin (Table 2). 
The CD11d antibody, a specific marker for macrophages, was positive and predominant in neoplastic cells from samples sent from the spleen, lung and lymph node. The other antibodies were made only in the spleen sample, the HLA-DR marker of the main histocompatibility complex MHC II, the CD18 marker of histiocytic neoplasms and the CD163 marker of histiocytic neoplas$\mathrm{ms}$ and leukemias were positive in the organ's neoplastic cells, but with less prevalence in macrophages, since its main function is phagocytosis and not the presentation of antigens as in histiocytic disorders of dendritic cells. The CD20 antibody of lymphoid lineage B and the CD3 receptor of epsilon $\mathrm{T}$ lymphocytes (both polyclonal) were also performed, being negative in the neoplastic cells of the spleen. Immunohistochemistry is essential to differentiate it from other round cell tumors, such as lymphomas, poorly differentiated mastocytomas and other histiocytic disorders, as a lymphocytic inflammatory infiltrate can be observed [6].

It can be concluded that HHS is a neoplasm with nonspecific clinical signs, rapid development and poor prognosis, and complementary exams are extremely important for the diagnostic conclusion. Thus, in the present case, the cytological examination was a screening method and the histopathology and immunohistochemistry tests were essential to obtain the definitive diagnosis, with the CD11d marker being specific for the diagnostic confirmation of HHS.

\section{MANUFACTURERS \\ ${ }^{1}$ Ouro Fino Saúde Animal. Cravinhos, SP, Brazil \\ ${ }^{2}$ Sanofi Medley Farmacêutica Ltda. Suzano. SP, Brazil. \\ ${ }^{3}$ Dako North America Inc. Carpinteria, CA, USA.}

Declaration of interest. The authors report no conflicts of interest. The authors alone are responsible for the content and writing of this paper.

\section{REFERENCES}

1 Affolter V.K. \& Moore P.F. 2002. Localized and Disseminated Histiocytic Sarcoma of Dendritic Cell Origin in Dogs. Veterinary Pathology. 39: 74-83. DOI: 10.1354/vp.39-1-74

2 Almeida A.R.C. 2017. Doenças Histiocíticas no Cão - Descrição de Casos Clínicos. 71f. Portugal. Dissertação (Mestrado em Medicina Veterinária). Universidade de Trás-Os-Montes e Alto Douro, Vila Real.

3 Constantino-Casas F., Mayhew D., Hoather T.M. \& Dobson J.M. 2011. The Clinical Presentation and HistopathologicImmunohistochemical Classification of Histiocytic Sarcomas in the Flat Coated Retriever. Veterinary Pathology. 48: 764-771. DOI: $10.1177 / 0300985810385153$

4 Jark P.C. \& Rodigheri S.M. 2016. Distúrbios Histiocíticos. In: Daleck C.R. \& De Nardi A.B. (Eds). Oncologia em Cães e Gatos. 2.ed. Rio de Janeiro: Roca, pp.972-990.

5 Friendrichs K.R. \& Young K.M. 2008. Histiocytic sarcoma of macrophage origin in a cat: case report with a literature review of feline histiocytic malignancies and comparison with canine hemophagocytic histiocytic sarcoma. Veterinary Clinical Pathology. 37: 121-128. DOI: 10.1111/j.1939-165X.2008.00005.x.

6 Fulmer A.K. \& Mauldin G.E. 2007. Canine histiocytic neoplasia: An overview. Canadian Veterinary Journal. 48 : 1041-1050.

7 Hedan B., Thomas R., Motsinger-Reif A., Abadie J., Andre C., Cullen J. \& Breen M. 2011. Molecular cytogenetic characterization of canine histiocytic sarcoma: A spontaneous model for human histiocityc cancer identifies deletion of tumor suppressor genes and highlights influence of genetic background on tumor behavior. BMC Cancer. 11: 1-14. DOI: 10.1186/1471-2407-11-201.

8 Liao A.T., McMahon M. \& London C.A. 2006. Identification of a novel germline MET mutation in dogs. Animal Genetics. 37: 248-252. DOI: 10.1111/j.1365-2052.2006.01415.x

9 Masuda E.K., Anjos B.L., Trost M.E., Oliveira Filho J.C., Gabriel A.L., Irigoyen L.F. \& Fighera R.A. 2009. Sarcoma histiocítico disseminado em um cão. Clínica Veterinária. 79: 44-46.

10 Moore P.F., Affolter V.K. \& Vernau W. 2006. Canine Hemophagocytic Histiocytic Sarcoma: A Proliferative Disorder of CD11D+ Macrophages. Veterinary Pathology. 43: 632-645. DOI: 10.1354/vp.43-5-632.

11 Teixeira L.V., Martins D.B., França R.T., Amaral A.S., Mazzantin C.M. \& Lopes S.T.A. 2012. Hemophagocytic histiocytic sarcoma in feline. Ciência Rural. 42: 675-678. DOI: 10.1590/S0103-84782012000400016. 